\title{
Prediabetes: a position statement from the Australian Diabetes Society and Australian Diabetes Educators Association
}

\author{
Stephen M Twigg, Maarten C Kamp, Timothy M Davis, Elizabeth K Neylon and Jeffrey R Flack
}

$\longrightarrow$

onditions in which blood glucose levels are elevated but not in the range of diabetes mellitus occur commonly. The term "prediabetes" has recently been adopted internationally to describe many of these conditions, but no national or international management guidelines have been published. This position statement has been developed as outlined in Box 1 to provide consensus-based clinical care guidelines for patients with prediabetes.

\section{Definitions}

In 2002, the American Diabetes Association and the United States Department of Health and Human Services ${ }^{2}$ defined prediabetes as the condition in which blood glucose levels are elevated above the normal range but do not satisfy the criteria for the diagnosis of diabetes mellitus, ${ }^{3}$ defined by the World Health Organization. ${ }^{4}$ For practical purposes, only a single plasma glucose measurement in the defined category, rather than two on separate days, is required to diagnose prediabetes, with testing done in the absence of severe metabolic stress or illness.

With current usage, prediabetes can be applied as a label for a disorder or as a risk category for diabetes and cardiovascular disease. There is no clear evidence which of these has the greater clinical utility. In addition, even within the established biochemical ranges for impaired fasting glucose/glycaemia (IFG) and impaired glucose tolerance (IGT), in most populations there is no glycaemic threshold for the risk of prospective diabetes, cardiovascular disease, or all-cause mortality. ${ }^{5}$ The single category of prediabetes thus incorporates a continuum of "cardiometabolic risk".

Although the American Diabetes Association has recently defined IFG as a fasting plasma glucose level of $5.6-6.9 \mathrm{mmol} / \mathrm{L},{ }^{5}$ this has not been adopted in this position statement. There is evidence that such a low threshold glucose level may cause the IFG category to lose specificity and positive predictive value as a risk factor for diabetes. ${ }^{6}$ Thus, the biochemical range used for IFG is the WHO definition of $\geqslant 6.1 \mathrm{mmol} / \mathrm{L}$ and $<7.0 \mathrm{mmol} / \mathrm{L}($ Box 2$){ }^{4}$

\section{Prevalence, pathogenesis and detection}

In the AusDiab study, a cross-sectional survey of adults aged 25 years and older, ${ }^{7}$ IGT affected $10.6 \%$ of subjects, being more common in women ( $11.9 \% \mathrm{v} 9.2 \%$ in men), and IFG was present in $5.8 \%$, being more prevalent in men ( $8.1 \% \mathrm{v} 3.4 \%$ in women). This represents an overall prediabetes prevalence of $16.4 \%$ in Australian adults $(\geqslant 25$ years). ${ }^{7}$

As in type 2 diabetes, the pathogenesis of prediabetes is linked to relative insulin deficiency and tissue insulin resistance causing abnormal blood glucose levels despite secondary hyperinsulinaemia. ${ }^{8}$ A recent review suggests that IFG is associated with hepatic insulin resistance, resulting in fasting hyperglycaemia, and IGT is associated predominantly with skeletal muscle insulin resistance. ${ }^{8}$

Although, intuitively, risk factors for prediabetes may mirror those of type 2 diabetes, no prospective studies have comprehensively identified risk factors for prediabetes development. A study of premenopausal women found that obesity and waist circumference were associated with prediabetes, ${ }^{9}$ and other cross-sectional studies have

\section{ABSTRACT}

- Prediabetes, the presence of impaired fasting glucose/ glycaemia and/or impaired glucose tolerance, affects about $16.4 \%$ of Australian adults.

- People with prediabetes are at increased risk of developing diabetes, and cardiovascular and other macrovascular disease.

- Management includes reducing cardiovascular disease risk factors, specifically lipid and blood pressure abnormalities, and smoking-cessation counselling. To help prevent progression to diabetes, people with prediabetes who are overweight or obese require intensive lifestyle intervention. Medication to help prevent diabetes may also be used, but only after a minimum of 6 months of lifestyle intervention.

- In people with prediabetes, there is no role for routinely testing: capillary blood glucose; glycated haemoglobin $\left(\mathrm{HbA}_{1 \mathrm{c}}\right)$ levels; serum insulin or pancreatic C-peptide levels; or testing for ischaemic heart disease or the microvascular complications of diabetes.

- Follow-up assessment of glycaemia in prediabetes requires a formal $75 \mathrm{~g}$ oral glucose tolerance test, initially performed annually, with subsequent individualised testing frequency.

MJA 2007; 186: 461-465

shown a positive association of prediabetes with increasing age, particularly for IGT. ${ }^{7}$ On the basis of the similar cardiovascular profiles of prediabetes and diabetes, ${ }^{10}$ the clinical risk factors for prediabetes have been described as those for type 2 diabetes. ${ }^{11}$

Prediabetes is often an incidental finding in people who are undergoing biochemical testing for diabetes. As in type 2 diabetes, ${ }^{12}$ screening for prediabetes among adults who are overweight, using a stepped approach of a fasting plasma glucose (FPG) measurement then, if indicated, a $75 \mathrm{~g}$ oral glucose tolerance test (OGTT), appears more cost-effective than a $75 \mathrm{~g}$ OGTT as a first-line investigation, especially in screening the general community. ${ }^{13}$

No prospective data are available to determine whether screening for the presence of prediabetes gives long-term health benefits. Thus, currently, because of the lack of fully defined risk factors for prediabetes and the lack of longer-term intervention data, screening specifically for prediabetes is not recommended. We acknowledge that it will occur, in association with biochemical screening for diabetes. ${ }^{12}$ According to current guidelines, ${ }^{12}$ if in screening for type 2 diabetes, a formal laboratory FPG measurement is between 5.5 and $6.9 \mathrm{mmol} / \mathrm{L}$, then a formal $75 \mathrm{~g}$ OGTT should be performed to exclude diabetes.

\section{Clinical significance of prediabetes}

\section{Diabetes risk}

Given the natural history of prediabetes, about 3\%-10\% of people per year with prediabetes develop diabetes. Data are particularly well substantiated for IGT. In the Diabetes Prevention Program, ${ }^{14}$ with subjects who had IGT, with or without IFG, there was about a $10 \%$ 


\section{Consensus process and methods}

Aim: To develop recommendations for the clinical management of prediabetes for physicians and allied health care professionals.

Source: The Prediabetes Working Party, formed in late 2004, comprises representatives from the Australian Diabetes Society (ADS) Council and the Australian Diabetes Educators Association (ADEA) Board.

Methods: A review of peer-reviewed journals was conducted using MEDLINE (1966 - 30 September 2005). To provide a historical profile to the term "prediabetes" and a more complete approach to therapy, specific key relevant references outside of these dates were also included. Relevant articles were identified using the subject headings "glucose intolerance" and "prediabetes". To ensure all appropriate articles were identified, the text words "IGT", "IFG", "impaired glucose tolerance", "impaired fasting glucose", and "impaired fasting glycemia" were used.

Levels of evidence: Articles retrieved were graded according to their level of evidence (based on the National Health and Medical Research Council [NHMRC] levels of evidence [I, II, III (including III-1, III-2, III-3), and IV] designated E1, E2, E3, and E4, respectively). ${ }^{1}$ When an NHMRC level of evidence for a clinically relevant aspect of prediabetes management was lacking, consensus expert opinion of the Prediabetes Working Party (designated E5) was applied.

Final recommendations: Comments from members of the ADS Council and ADEA Board on the draft position statement were received and considered. Final clinical recommendations were then prepared by the Prediabetes Working Party and approved by the ADS Council and ADEA Board.

annual rate of progression to diabetes in the control group. Other studies have shown similar or somewhat lower rates for progression from IGT or IFG to diabetes. ${ }^{15,16}$ The combination of IFG and IGT confers a greater risk of diabetes than either category alone. Overall, prediabetes confers about a sixfold increased risk of diabetes compared with normal glucose tolerance.

In most populations studied, the rates of conversion from IFG and IGT to diabetes are similar, with IGT having greater sensitivity ${ }^{17}$ but less specificity ${ }^{18}$ than IFG in predicting diabetes risk. Each category has a similar positive predictive value. ${ }^{5}$ In an 11 -year follow-up study among adults with IGT in Mauritius, 46\% developed diabetes, 28\% remained unchanged in category, $4 \%$ developed IFG, and glucose levels normalised in 24\%. Among adults with IFG, 38\% developed diabetes, $7 \%$ remained unchanged, $17 \%$ developed IGT, and glucose levels normalised in $38 \% .{ }^{18}$ Thus, many people with prediabetes (a quarter or more) may revert long term to having normal glucose tolerance, and after a protracted follow-up, only about $50 \%$ of people with IGT or IFG will develop diabetes.

\section{Cardiovascular disease risk}

In comparison with adults who have normal glucose tolerance, people with prediabetes have an increased risk of developing cardiovascular disease (CVD) and cardiovascular and all-cause mortality. ${ }^{19-21}$ There is a two- to threefold increased prospective risk of cardiovascular events, ${ }^{13}$ which is most marked in younger adults with prediabetes. ${ }^{22}$ These rates for cardiovascular events approach those in people who have type 2 diabetes. ${ }^{23}$ Prediabetes is associated with increased rates of the cardiovascular risk factors found in people with type 2 diabetes. ${ }^{21}$ In Australia, increased serum triglyceride levels, decreased high-density lipoprotein (HDL) cholesterol levels, hypertension and central adiposity are more common in adults with prediabetes com- pared with those with normal glucose tolerance, but less so than in people with type 2 diabetes. $^{7}$

Some data indicate that people with IGT and normal levels of fasting plasma glucose have a greater risk of CVD than those with IFG. ${ }^{19,21}$ In addition, when other known CVD risk factors, such as hypertension and lipid abnormalities, are adjusted for statistically, IGT, but possibly not IFG, remains as an independent CVD risk factor. ${ }^{20}$ An increasing plasma glucose level in IGT is associated with a greater risk of cardiovascular death. ${ }^{20}$

\section{Associations with the metabolic syndrome}

The metabolic syndrome (MetS) refers to a clustering in an individual of CVD risk factors and diabetes susceptibility. ${ }^{24}$ People with MetS have about a twofold increased risk of developing diabetes and cardiovascular disease, compared with those without the syndrome. ${ }^{25}$ Several MetS definitions exist, with two being widely used. ${ }^{26,27}$ Recently, a third definition has been adopted by the International Diabetes Federation. ${ }^{28}$ Each definition has impairment of glucose metabolism as an optional criterion, although some consider only IFG. Most adults who have prediabetes will also have MetS. Whether prediabetes or MetS best defines diabetes and cardiovascular risk remains to be determined.

\section{Management of prediabetes}

The management of prediabetes is determined by the increased risk of developing both diabetes and cardiovascular disease. ${ }^{19}$ Diabetes prevention studies in people with prediabetes have been conducted. In contrast, prevention of subsequent cardiovascular complications in prediabetes has not been studied specifically.

\section{Lifestyle intervention}

Several randomised, prospective studies of subjects with prediabetes have documented beneficial effects of lifestyle intervention in preventing type 2 diabetes. In the Diabetes Prevention Program ${ }^{14}$ targets in the lifestyle intervention arm were weight loss of $7 \%$ and moderate physical activity (such as brisk walking) for a total of 150 min weekly. Although most subjects did not achieve these goals, an average of $6 \%$ $(5.6 \mathrm{~kg})$ of body weight was lost and $57 \%$ of subjects undertook the targeted amount of physical activity. After an average follow-up of 2.8 years, there was a $58 \%$ relative risk reduction in progression to diabetes in the lifestyle-intervention group compared with the controls. By the end of the study, while most of the weight lost in the intervention arm

\section{Criteria for diagnosing prediabetes - impaired fasting glucose (IFG) level and impaired glucose tolerance $(\text { IGT })^{4}$}

\begin{tabular}{lc} 
Prediabetes (IFG or IGT) & $\begin{array}{c}\text { Plasma glucose level } \\
\text { (mmol/L) }\end{array}$ \\
\hline IFG & $\geqslant 6.1$ \\
Fasting & $<7.8$ \\
and 2-hour post-glucose load* & \\
(if measured) & $<7.0$ \\
IGT & $\geqslant 7.8$ and $<11.1$ \\
Fasting & \\
and 2-hour post-glucose load* & \\
\hline${ }^{*}$ A standardised 75 g oral glucose tolerance test.
\end{tabular}




\section{POSITION STATEMENT}

had been regained, the approximately fivefold difference in physical activity levels between the groups was largely maintained.

Similarly, in the Finnish Diabetes Prevention Study, ${ }^{15}$ after 3.2 years of follow-up, there was a $58 \%$ relative risk reduction in the incidence of diabetes in the lifestyle intervention group compared with controls. Those who achieved the greatest number of the five pre-established lifestyle goals in the study (weight reduction $>5 \%$; fat intake $<30 \%$ of total energy intake; saturated fat intake $<10 \%$ of total energy intake; dietary fibre intake $\geqslant 15 \mathrm{~g} / 1000 \mathrm{kcal}$; and at least moderate intensity exercise for $>4$ hours weekly) showed the lowest rate of diabetes development. The Da Qing IGT and Diabetes Study ${ }^{16}$ of adults with prediabetes compared a control group with three treatment groups: diet alone, exercise alone, or diet plus exercise. Over 6 years, the relative risk reduction in progression to diabetes was $31 \%$ in the diet group, $46 \%$ in the exercise group, and $42 \%$ in the combination group.

Two other recent randomised clinical trials have also indicated that lifestyle intervention is effective in reducing the incidence of diabetes in subjects with prediabetes from different ethnic groups. In 458 Japanese men with IGT, the cumulative 4-year incidence of diabetes was $9.3 \%$ in the lifestyle control group, versus $3.0 \%$ in the intensive intervention group, with a relative reduction in development of diabetes of $67 \% .^{29}$ In the Indian Diabetes Prevention Programme, lifestyle modification in 531 subjects with IGT at enrolment delayed the development of type 2 diabetes in Asian Indian subjects, with a 3year cumulative incidence of diabetes of $55 \%$ in the control group compared with $39 \%$ in the lifestyle intervention group. ${ }^{30}$ The relative risk reduction of $29 \%$ with lifestyle modification was similar to that in a parallel group treated with both metformin and lifestyle intervention.

Sustained moderate loss of body weight in people with prediabetes is an important predictor of a positive outcome of lifestyle intervention. It is difficult to dissect out the role that macronutrients played in protecting against development of diabetes, although most of the intervention studies did focus on reducing total energy intake from fat, including reducing saturated fat intake, and increasing dietary fibre intake. By contrast, at present there is insufficient information to determine whether reducing dietary total carbohydrate intake, or glycaemic index or load, will protect against the development of diabetes. ${ }^{31}$ Factors other than a reduction in body weight are likely to have retarded the development of diabetes, potentially through improvements in insulin sensitivity or reduction in central body fat. ${ }^{30}$ Physical activity may work through mechanisms involving improved insulin sensitivity and weight-loss maintenance.

\section{Health care delivery}

In the Finnish Diabetes Prevention Study and the Diabetes Prevention Program, multidisciplinary teams included a physician, dietitian, nurse, psychologist and a physiotherapist. In each of the studies, there was expertise in nutrition, physical activity and behavioural change. Individual patient education and coaching was used to implement intensive lifestyle change. The economic cost of implementing such an approach in routine clinical care in people with prediabetes may not be justifiable, ${ }^{32}$ and it has been proposed that an emphasis on group interventions may provide a more cost-effective approach, although this has not been formally tested. ${ }^{33}$ Cost-efficient, practical health care delivery models for the lifestyle aspects of diabetes prevention require further study. In the interim, methods that lead to sustained improvements in nutrition and physical activity levels should be encouraged.

\section{Pharmacotherapy}

Multiple medications have been shown in randomised, doubleblinded, prospective studies to reduce the incidence of diabetes in people with prediabetes. In each of these studies, patients in all intervention arms commonly received general healthy lifestyle advice in addition to active medication or placebo. In the Diabetes Prevention Program, subjects allocated at random to metformin therapy, $850 \mathrm{mg}$ twice daily, showed a $31 \%$ risk reduction in progression to diabetes compared with the control group. ${ }^{14}$ Younger age ( $<60$ years) and overweight predicted greatest benefit. Adherence to metformin was about $70 \%$, compared with $75 \%$ for placebo. When metformin was stopped, its benefit in preventing diabetes was maintained in about $83 \%$ of subjects. The combined effect of intensive lifestyle intervention and metformin therapy was not studied in the Diabetes Prevention Program.

In the STOP-NIDDM trial, the glucosidase inhibitor acarbose ( $100 \mathrm{mg}$ three times daily) reduced progession to diabetes by about $25 \%$ after 3.3 years. ${ }^{34}$ Acarbose may also reduce the occurrence of cardiovascular events, ${ }^{34}$ but this finding needs to be confirmed in other studies. ${ }^{35}$ In the TRIPOD study, ${ }^{36}$ women with a history of gestational diabetes (a condition predisposing to diabetes) were less likely to develop diabetes when treated with troglitazone (an agent subsequently withdrawn because of idiosyncratic liver dysfunction). This positive finding is likely to be a class effect of the thiazolidinedione group of medications, particularly as the recent DREAM study showed that rosiglitazone, $8 \mathrm{mg}$ daily, taken for a median of 3 years, reduced the risk of diabetes or death by $60 \%$ in subjects with prediabetes. $^{37}$ In the XENDOS study, ${ }^{38}$ the gastrointestinal lipase inhibitor orlistat taken three times daily reduced diabetes risk by $37 \%$ over 4 years in obese adults with prediabetes.

None of these medications is approved for use in people with prediabetes on the Pharmaceutical Benefits Scheme. In addition, considering the beneficial effects of diet and exercise, in general, medication cannot be recommended in preference to lifestyle intervention in preventing diabetes. It is suggested that a minimum 6 months of lifestyle intervention be trialled before pharmacotherapy is commenced, as the nadir of weight loss occurred at this time in the Diabetes Prevention Program. ${ }^{14}$ Definitions of failure would include weight gain rather than weight loss and no evidence of increased physical activity or achievement of recommended macronutrient dietary change.

\section{Managing macrovascular risk and screening for end-organ complications}

In prediabetes, for people without known cardiovascular disease, no targets have been established for ischaemic heart disease risk factors. Because people with prediabetes have a similar overall cardiovascular risk to those with type 2 diabetes, we recommend that macrovascular treatment targets for adults with prediabetes and diabetes should be the same. Thus, blood pressure targets should generally be <130/ $80 \mathrm{mmHg} .{ }^{39}$ For individuals without a known history of macrovascular disease, lipid targets in prediabetes should be in line with National Heart Foundation of Australia guidelines ${ }^{40}$ for primary prevention (total cholesterol, <4.0 mmol/L; HDL cholesterol, > $1.0 \mathrm{mmol} / \mathrm{L}$; triglycerides, $<1.7 \mathrm{mmol} / \mathrm{L}$; and calculated low-density lipoprotein (LDL) cholesterol, $<2.5 \mathrm{mmol} / \mathrm{L}){ }^{41}$ Antiplatelet therapy for cardiovascular prophylaxis in prediabetes is the same as recommended in type 2 diabetes. Cigarette smokers should be counselled to stop smoking. 


\section{POSITION STATEMENT}

\section{Consensus clinical recommendations - summary}

Definition and detection: "Prediabetes" is defined as the presence of impaired fasting glucose (IFG) and/or impaired glucose tolerance (IGT). A single abnormal reading at formal testing is adequate to define prediabetes. People who have prediabetes are at increased risk of developing diabetes, although a proportion of those with prediabetes can revert to normal glucose tolerance. Prediabetes may be incidentally detected when screening for diabetes. There is no current proven clinical role for specifically screening for prediabetes. This is consistent with current National Health and Medical Research Council guidelines for screening for diabetes (E5). Reducing cardiovascular risk: People with prediabetes are at increased risk of developing cardiovascular and other macrovascular disease (E2). Assessment and management of risk factors for cardiovascular disease, specifically lipid and blood pressure abnormalities, should be undertaken. Although there have been no intervention studies specific to prediabetes, the blood pressure and lipid targets suggested are equivalent to those for type 2 diabetes, as is the use of prophylactic antiplatelet therapy (E5).

Preventing progression to diabetes: The appropriate intervention for people with prediabetes who are overweight or obese should be intensive lifestyle intervention (E1), aiming to achieve: a minimum of $5 \%-7 \%$ body weight loss, with reduced total fat and saturated fat intake to $<30 \%$ and $<10 \%$ of total daily calories, respectively; optimal dietary fibre intake; and 150 minutes of physical activity per week (E2). The multidisciplinary approach is the treatment method with strongest evidence (E2). It is recommended that a minimum 6 months of lifestyle intervention be trialled before pharmacotherapy is considered (E5). Pharmacotherapy may particularly involve metformin, ${ }^{*}$ (850 mg twice daily), which appears to be relatively more efficacious in younger ( $<60$ years) and more overweight people (E2). Other options include orlistat* $(120 \mathrm{mg}$ three times daily); acarbose* (100 mg three times daily); or a thiazolidinedione* (eg, rosiglitazone $8 \mathrm{mg}$ daily or pioglitazone) (E2).

Testing generally not required: In the absence of specific clinical indications in an individual, there is no role for routinely conducting the following tests in people who have prediabetes: capillary blood glucose measurement, using a home blood glucose meter; glycated haemoglobin $\left(\mathrm{HbA}_{1 \mathrm{c}}\right)$ level; serum insulin or pancreatic $\mathrm{C}$-peptide levels; tests for ischaemic heart disease (if there is no clinical evidence for the condition); tests for microvascular complications of diabetes, such as retinopathy screening or neuropathy assessment; or tests for microalbuminuria or proteinuria (E5).

Follow-up: Follow-up testing of glycaemia in prediabetes requires a formal $75 \mathrm{~g}$ oral glucose tolerance test, initially performed annually. In subsequent years, the frequency of this test can be individualised, with retesting at intervals of $1-3$ years (E5).

* Note that none of the medications mentioned is approved for management of prediabetes on the Pharmaceutical Benefits Scheme. The medication doses given are based on studies of prevention of diabetes in people with impaired glucose tolerance. Most of these studies have not been replicated to date and in at least one study the validity of the methodology remains questionable.

Hence, lifestyle intervention to prevent diabetes and help reduce

cardiovascular risk should be preferentially emphasised (E5).

\section{Monitoring}

\section{Tests for glycaemia}

By definition, people with prediabetes have relatively mild and less marked elevations in blood glucose levels compared with those with diabetes. Particularly as capillary whole-blood glucose measurements using portable monitors have significant attendant error $(>10 \%),{ }^{42}$ there is no role for routine home capillary blood glucose monitoring in people with prediabetes. No data exist to define the utility of measurement of glycated haemoglobin $\left(\mathrm{HbA}_{\mathrm{lc}}\right)$ in monitoring prediabetes. In addition, serum insulin and pancreatic $\mathrm{C}$-peptide levels have no established clinical role in prediabetes.

\section{Screening for end-organ complications}

People with prediabetes who are asymptomatic for ischaemic heart disease should be assessed individually, and a history of symptoms and macrovascular risk factors obtained before a decision is made to investigate for possible myocardial ischaemia.

FPG and 2-hour levels post-glucose load are associated with retinopathy and nephropathy, with approximate thresholds near or below the current diagnostic criteria for diabetes. ${ }^{43}$ Thus, only rarely do people with prediabetes develop these microvascular complications. ${ }^{43} \mathrm{~A}$ painful form of neuropathy, which tends to be transient, may also rarely develop in prediabetes. ${ }^{44}$ However, no evidence exists to indicate that routine screening for microvascular disease in prediabetes affects clinical outcome.

\section{Glycaemia reassessment at follow-up}

The appropriate interval for retesting of adults with prediabetes using a $75 \mathrm{~g}$ OGTT is arbitrary. Initially, 12 months may be appropriate, consistent with current WHO guidelines for diabetes screening, especially to assess rate of change of abnormal glucose tolerance. ${ }^{4}$ If a particular patient develops factors in the interim that increase the risk of developing diabetes, such as significant weight gain, then earlier testing may be indicated. After the initial 12-month follow-up, with a $75 \mathrm{~g}$ OGTT, if the results do not show deterioration in glycaemia, the retesting interval could be increased, for example to 2 or 3 years.

Box 3 summarises the clinical recommendations for prediabetes.

\section{Acknowledgements}

We thank the Project Officer, Dr Abdullah Omari (ADS paid consultant), and the ADS Council members who were not part of the Prediabetes Working Party for their contribution: Dr Wah Cheung, Dr Alicia Jenkins, Professor Mark Febbraio, Dr Terri Allen, and Associate Professor Glenn Ward. The ADS members who commented on the draft statement are also recognised, as is the contribution of the ADEA Board, especially Ms Shirley Cornelius. Assistance from ADS Secretary, Ms Suzie Neylon, and the Executive Officer of the Australian Diabetes Professional Organisations, Mr Chris Thorpe, is acknowledged with thanks.

\section{Competing interests}

Stephen Twigg has received speaker fees for educational meetings in diabetes, from Alphapharm, Eli Lilly, GlaxoSmithKline, Novo Nordisk, Sanofi-aventis and Servier.

\section{Author details}

Stephen M Twigg, MBBS, FRACP, PhD, Associate Professor, ${ }^{1}$ Endocrinologist ${ }^{2}$

Maarten C Kamp, FRACP, MHA, Senior Endocrinologist, ${ }^{3}$ Associate Professor $^{4}$

Timothy M Davis, FRACP, Diabetologist, General Physician, ${ }^{5}$ Professor of Medicine ${ }^{6}$

Elizabeth K Neylon, DAA, CDE, Dietitian, Area-Wide Diabetes Education Coordinator $^{7}$

Jeffrey R Flack, FRACP, MMed, Senior Staff Endocrinologist, Director ${ }^{8}$ Clinical Associate Professor?

1 Department of Medicine, University of Sydney, Sydney, NSW.

2 Royal Prince Alfred Hospital, Sydney, NSW.

3 Gold Coast Hospital, Gold Coast, QLD.

4 Griffith University, Brisbane, QLD.

5 Fremantle Hospital, Fremantle, WA.

6 School of Medicine and Pharmacology, University of Western Australia,

Fremantle, WA.

7 South Metropolitan Health Service Area, Department of Health, Perth, WA 


\section{POSITION STATEMENT}

8 Diabetes Centre, Bankstown-Lidcombe Hospital, Sydney, NSW.

9 University of New South Wales, Sydney, NSW.

Correspondence: stwigg@med.usyd.edu.au

\section{References}

1 National Health and Medical Research Council. A guide to the development, implementation and evaluation of clinical practice guidelines. Canberra: NHMRC, 1999.

2 US Department of Health and Human Services, American Diabetes Association. HHS, ADA warn Americans of "pre-diabetes". Press release. 27 Mar 2002. Washington, DC: DHHS, 2002. http://www.hhs.gov/news/press/2002pres/ 20020327.html (accessed Mar 2007).

3 American Diabetes Association. Standards of medical care in diabetes. Diabetes Care 2005; 28 Suppl 1: S4-S36.

4 World Health Organization. Definition, diagnosis and classification of diabetes mellitus and its complications: report of a WHO consultation. Part 1: diagnosis and classification of diabetes mellitus. Geneva: WHO, 1999. http://unw. diabetes.com.au/pdf/who_report.pdf (accessed Mar 2007).

5 Genuth S, Alberti KG, Bennett P, et al; Expert Committee on the Diagnosis and Classification of Diabetes Mellitus. Follow-up report on the diagnosis of diabetes mellitus. Diabetes Care 2003; 26: 3160-3167.

6 Vaccaro O, Riccardi G. Changing the definition of impaired fasting glucose: impact on the classification of individuals and risk definition. Diabetes Care 2005; 28: $1786-1788$

7 Dunstan DW, Zimmet PZ, Welborn TA, et al. The rising prevalence of diabetes and impaired glucose tolerance: the Australian Diabetes, Obesity and Lifestyle Study. Diabetes Care 2002; 25: 829-834.

8 Abdul-Ghani MA, Tripathy D, DeFronzo RA. Contributions of beta-cell dysfunction and insulin resistance to the pathogenesis of impaired glucose tolerance and impaired fasting glucose. Diabetes Care 2006; 29: 1130-1139.

9 Piche ME, Despres JP, Pascot A, et al. Impaired fasting glucose vs. glucose intolerance in pre-menopausal women: distinct metabolic entities and cardiovascular disease risk? Diabetic Med 2004; 21: 730-737.

10 Henry $\mathrm{P}$, Thomas F, Benetos A, et al. Impaired fasting glucose, blood pressure and cardiovascular disease mortality. Hypertension 2002; 40: 458-463.

11 American Diabetes Association. Screening for type 2 diabetes. Diabetes Care 2004; 27 Suppl 1: S11-S14.

12 Report of the Expert Committee on the Diagnosis and Classification of Diabetes Mellitus. Diabetes Care 1997; 20: 1183-1197.

13 Zhang P, Engelgau MM, Valdez R, et al. Costs of screening for pre-diabetes among US adults: a comparison of different screening strategies. Diabetes Care 2003; 26: 2536-2542.

14 Knowler WC, Barrett-Connor E, Fowler SE, et al; Diabetes Prevention Program Research Group. Reduction in the incidence of type 2 diabetes with lifestyle intervention or metformin. N Engl J Med 2002; 346: 393-403.

15 Tuomilehto J, Lindstrom J, Eriksson JG, et al; Finnish Diabetes Prevention Study Group. Prevention of type 2 diabetes mellitus by changes in lifestyle among subjects with impaired glucose tolerance. N Engl J Med 2001; 344: 1343-1350.

16 Pan XR, Li GW, Hu YH, et al. Effects of diet and exercise in preventing NIDDM in people with impaired glucose tolerance. The Da Qing IGT and Diabetes Study. Diabetes Care 1997; 20: 537-544.

17 Unwin N, Shaw J, Zimmet P, Alberti KG. Impaired glucose tolerance and impaired fasting glycaemia: the current status on definition and intervention. Diabet Med 2002; 19: 708-723

18 Shaw JE, Zimmet PZ, de Courten M, et al. Impaired fasting glucose or impaired glucose tolerance. What best predicts future diabetes in Mauritius? Diabetes Care 1999; 22: 399-402.

19 Decode Study Group. Glucose tolerance and cardiovascular mortality: comparison of fasting and 2-hour diagnostic criteria. Arch Intern Med 2001; 161: 397-405.

20 Coutinho M, Gerstein $\mathrm{HC}$, Wang $\mathrm{Y}$, et al. The relationship between glucose and incident cardiovascular events. A metaregression analysis of published data from 20 studies of 95,783 individuals followed for 12.4 years. Diabetes Care 1999; 22 : 233-240.

21 Liao D, Shofer JB, Boyko EJ, et al. Abnormal glucose tolerance and increased risk for cardiovascular disease in Japanese-Americans with normal fasting glucose. Diabetes Care 2001; 24: 39-44.

22 Bjornholt JV, Erikssen G, Aaser E, et al. Fasting blood glucose: an underestimated risk factor for cardiovascular death. Results from a 22-year follow-up of healthy nondiabetic men. Diabetes Care 1999; 22: 45-49.

23 Wood D, De Backer G, Faergeman O, et al. Prevention of coronary heart disease in clinical practice: recommendations of the Second Joint Task Force of European and other Societies on Coronary Prevention. Atherosclerosis 1998; 140: 199-270.
24 Lempiainen $\mathrm{P}$, Mykkanen L, Pyorala K, et al. Insulin resistance syndrome predicts coronary heart disease events in elderly nondiabetic men. Circulation 1999; 100: 123-128.

25 Laaksonen DE, Lakka HM, Niskanen LK, et al. Metabolic syndrome and development of diabetes mellitus: application and validation of recently suggested definitions of the metabolic syndrome in a prospective cohort study. Am J Epidemiol 2002; 156: 1070-1077.

26 Alberti K, Zimmet P. Definition, diagnosis and classification of diabetes mellitus and its complications. Part 1: diagnosis and classification of diabetes mellitus provisional report of a WHO consultation. Diabet Med 1998; 15: 539-553.

27 Expert Panel on Detection, Evaluation, and Treatment of High Blood Cholesterol in Adults. Executive Summary of the Third Report of the National Cholesterol Education Program (NCEP) Expert Panel on Detection, Evaluation, And Treatment of High Blood Cholesterol In Adults (Adult Treatment Panel III). JAMA 2001; 285: 2486-2497.

28 Alberti KG, Zimmet P, Shaw J; IDF Epidemiology Taskforce Consensus Group. The metabolic syndrome - a new worldwide definition. Lancet 2005; 366: 10591062.

29 Kosaka K, Noda M, Kuzuya T. Prevention of type 2 diabetes by lifestyle intervention: a Japanese trial in IGT males. Diabetes Res Clin Pract 2005; 67: 152-162.

30 Ramachandran A, Snehalatha C, Mary S, et al; Indian Diabetes Prevention Programme (IDPP). The Indian Diabetes Prevention Programme shows that lifestyle modification and metformin prevent type 2 diabetes in Asian Indian subjects with impaired glucose tolerance (IDPP-1). Diabetologia 2006; 49: 289297.

31 Sheard NF, Clark NG, Brand-Miller JC, et al. Dietary carbohydrate (amount and type) in the prevention and management of diabetes: a statement by the American Diabetes Association. Diabetes Care 2004; 27: 2266-2271.

32 Eddy DM, Schlessinger L, Kahn R. Clinical outcomes and cost-effectiveness of strategies for managing people at high risk for diabetes. Ann Intern Med 2005; 143: 251-264.

33 Lindstrom J, Louheranta A, Mannelin M, et al. The Finnish Diabetes Prevention Study (DPS): lifestyle intervention and 3-year results on diet and physical activity. Diabetes Care 2003; 26: 3230-3236.

34 Chiasson JL, Josse RG, Gomis R, et al. Acarbose for prevention of type 2 diabetes mellitus: the STOP-NIDDM randomised trial. Lancet 2002; 359: 20722077.

35 Van de Laar FA, Lucassen PL, Akkermans RP, et al. Alpha-glucosidase inhibitors for people with impaired glucose tolerance or impaired fasting blood glucose. Cochrane Database Syst Rev 2006; (4): CD005061.

36 Snitker S, Watanabe RM, Ani l, et al. Changes in insulin sensitivity in response to troglitazone do not differ between subjects with and without the common, functional Pro12Ala peroxisome proliferator-activated receptor-gamma2 gene variant: results from the Troglitazone in Prevention of Diabetes (TRIPOD) study. Diabetes Care 2004; 27: 1365-1368.

37 Gerstein HC, Yusuf S, Bosch J, et al; DREAM (Diabetes REduction Assessment with ramipril and rosiglitazone Medication) Trial Investigators. Effect of rosiglitazone on the frequency of diabetes in patients with impaired glucose tolerance or impaired fasting glucose: a randomised controlled trial. Lancet 2006; 368: 10961105

38 Torgerson JS, Hauptman J, Boldrin MN, Sjöström L. XENical in the prevention of diabetes in obese subjects (XENDOS) study: a randomized study of orlistat as an adjunct to lifestyle changes for the prevention of type 2 diabetes in obese patients. [Erratum appears in Diabetes Care 2004; 27: 856.] Diabetes Care 2004; 27: 155-161.

39 Chobanian AV, Bakris GL, Black HR, et al; National High Blood Pressure Education Program Coordinating Committee. The Seventh Report of the Joint National Committee on Prevention, Detection, Evaluation, and Treatment of High Blood Pressure: the JNC 7 report. JAMA 2003; 289: 2560-2572.

40 National Heart Foundation of Australia; Cardiac Society of Australia and New Zealand. Lipid management guidelines - 2001. Med J Aust 2001; 175 (9 Suppl): S57-S85. http://www.heartfoundation.com.au/downloads/Guideline_Lipid_Mgt_ 2001.pdf (accessed Mar 2007).

41 American Diabetes Association. Standards of medical care in diabetes - 2007. Diabetes Care 2007; 30 Suppl 1: S4-S41.

42 American Diabetes Association. Self-monitoring of blood glucose. Diabetes Care 1994; 17: 81-86.

43 Gabir MM, Hanson RL, Dabelea D, et al. Plasma glucose and prediction of microvascular disease and mortality: evaluation of 1997 American Diabetes Association and 1999 World Health Organization criteria for diagnosis of diabetes. Diabetes Care 2000; 23: 1113-1118.

44 Singleton JR, Smith AG, Bromberg MB. Increased prevalence of impaired glucose tolerance in patients with painful sensory neuropathy. Diabetes Care 2001; 24: 1448-1453.

(Received 13 Sep 2006, accepted 20 Feb 2007) 